\title{
SUMMABILITY OF HERMITE EXPANSIONS. I
}

\author{
S. THANGAVELU
}

\begin{abstract}
We study the summability of one-dimensional Hermite expansions. We prove that the critical index for the Riesz summability is $1 / 6$. We also prove analogues of the Fejér-Lebesgue theorem and Riemann's localisation principle.
\end{abstract}

\section{INTRODUCTION}

In 1965 Askey and Wainger [3] studied the mean convergence of Laguerre and Hermite expansions in $L^{p}(R)$. In their work it was proved that the series converges to the function if and only if $4 / 3<p<4$. Later in [17] Muckenhoupt enlarged the range of convergence by admitting more general weight functions. Since the series fails to converge for $p$ lying outside the interval $(4 / 3,4)$ it is necessary to consider suitable summability methods. In 1965 itself Freud and Knapowski [9] had solved the $(C, \alpha)$ summability problem for $p=\infty$ and $\alpha=1$. Here $(C, \alpha)$ stands for the Cesàro means of order $\alpha$. The $(C, 1)$ summability for $1 \leq p \leq \infty$ was established by E. L. Poiani in 1972 [21] and independently by Freud [7], also [8]. Both Freud and Poiani considered more general weight functions. For $0<\alpha<1$, the problem remained unsolved for some time.

In the 1980 s the $(C, \alpha)$ summability for $0<\alpha<1$ was taken up by $C$. Markett in a series of papers [14-16]. He obtained norm estimates from above and below for the $(C, \alpha)$ means of Hermite and Laguerre expansions and proved that they converge in the mean for $1 \leq p \leq \infty$ provided $\alpha$ is bigger than $1 / 2$. In the case of Laguerre series the problem was completely solved but it was not so in the Hermite case. The upper and lower bounds he obtained for the Hermite case match when $\alpha=0$ but for $0<\alpha<1 / 2$ there is a gap between them. Let $\alpha_{\text {cr }}$ denote the critical index, i.e. the largest $\alpha>0$ with the property that the expansion is not $(C, \alpha)$ summable for at least one $f$ in $L^{1}(R)$. Markett proved that $\alpha_{\mathrm{cr}}=1 / 2$ for the Laguerre expansions. But in the case of Hermite expansions he could only say that $1 / 6 \leq \alpha_{\mathrm{cr}} \leq 1 / 2$. It was noted that there is an essential difference between the two expansions.

Let $p_{0}$ be the largest $p$ in $[1,2]$ such that a given orthogonal expansion diverges for at least one $f$ in $L^{p_{0}}$ and let $\gamma$ be determined by $\left\|S_{n}\right\|_{1} \approx n^{\gamma}$ as

Received by the editors September 1, 1987.

1980 Mathematics Subject Classification (1985 Revision). Primary 42C10; Secondary 42C15.

Key words and phrases. Hermite expansion, summability, Riesz means, oscillatory integrals. 
$n$ tends to $\infty$ where $S_{n}$ are the partial sums operators. Thus for the Hermite series we have $p_{0}=4 / 3, \gamma=1 / 6$ and $1 / 6 \leq \alpha_{c r} \leq 1 / 2$ and for the Laguerre series $p_{0}=4 / 3, \gamma=1 / 2$ and $\alpha_{\text {cr }}=1 / 2$. In [13] Lorch conjectured that one should have $\gamma=\alpha_{c r}$ in both cases. This is certainly true for the Laguerre case and will be true for the Hermite case also if we have $\alpha_{\mathrm{cr}}=1 / 6$. At this point let us mention another principle noted by Askey in [1]. In several known cases it was noted that the line in the $(1 / p, \alpha)$ plane connecting the points $\left(1, \alpha_{\mathrm{cr}}\right)$ and $\left(1 / p_{0}, 0\right)$ always meets the point $(1 / 2,-1 / 2)$. This principle of Askey applies to the Laguerre case but would apply to the Hermite case only if $\alpha_{c r}=1 / 2$. Thus it appears that either Lorch's conjecture or Askey's principle fails in the Hermite case.

In this paper we consider the Riesz summability of the Hermite series for $0<\alpha<1 / 2$. The critical index turns out to be $1 / 6$ in accordance with the conjecture of Lorch. Thus Askey's principle fails in the Hermite case. We can summarize the main results of this paper as follows.

(i) Convergence in the norm. The Riesz means $S_{R}(\alpha)$ are uniformly bounded on $L^{p}, 1 \leq p \leq \infty$, provided $\alpha>1 / 6$. As $R$ tends to infinity $S_{R}(\alpha) f$ converges to $f$ in the norm for all $f$ in $L^{p}$.

(ii) Boundedness of the maximal operator. The maximal operator $M$ associated with $S_{R}(\alpha)$ is bounded on $L^{p}$, for $1<p<\infty$, and is weak type (1, 1) whenever $\alpha>1 / 6$. Consequently, the Riesz means $S_{R}(\alpha) f$ converges to $f$ a.e.

(iii) Analogue of the Fejer-Lebesgue theorem. If both $x$ and $-x$ are Lebesgue points of the function $f$, then $S_{R}(\alpha) f(x)$ converges to $f(x)$ as $R$ tends to infinity provided $\alpha>1 / 6$.

(iv) Analogue of Riemann's localisation theorem. If $f$ vanishes near the points $x$ and $-x$, then $S_{R}(\alpha) f(x)$ converges to 0 as $R$ tends to infinity under the condition that $\alpha>1 / 6$.

(v) Summability below the critical index. Assume that $0<\alpha<1 / 6$ and $f$ belongs to $L^{p}$. Then $S_{R}(\alpha) f$ converges to $f$ in the norm if and only if $4 /(6 \alpha+3)<p<4 /(1-6 \alpha)$.

All these results are proved in $\S 5$ as easy consequences of the estimates we get for the kernel of the Riesz means in $\S 4$. In the next section we begin with the preliminaries and obtain a good integral representation for the kernel of the Riesz means. In $\S 4$ we will study certain oscillatory integrals obtaining good estimates of them.

This paper represents a part of my Princeton University thesis written under the guidance of Professor E. M. Stein. I take this opportunity to express my gratitude towards my advisor for the constant encouragement and many useful suggestions I got from him during the course of this work. Some of my original proofs were simplified with great help from my advisor. 


\section{Preliminaries}

The following notations will be used. The Hermite polynomials $H_{n}(x)$ are defined by the equation $H_{n}(x)=(-1)^{n} \exp \left(x^{2}\right)(d / d x)^{n}\left\{\exp \left(-x^{2}\right)\right\}$. The normalised Hermite functions $\varphi_{n}(x)$ are then defined by

$$
\varphi_{n}(x)=\left(2^{n} \sqrt{\pi} n !\right)^{-1 / 2} \exp \left(-x^{2} / 2\right) H_{n}(x) .
$$

The functions $\left\{\varphi_{n}(x)\right\}$ form a complete orthonormal system in $L^{2}(-\infty, \infty)$. Since $\varphi_{n}(x)$ belongs to all $L^{p}$ spaces, we can talk about the generalised Fourier coefficients of any $f$ in $L^{p}$ for any $p$. The coefficients $f^{\wedge}(n)$ are defined by $f^{\wedge}(n)=\int f(x) \varphi_{n}(x) d x$. Thus to each function $f$ we have an associated expansion $f(x)=\sum_{n \geq 0} f^{\wedge}(n) \varphi_{n}(x)$. It is clear that the series converges to $f$ in the $L^{2}$ norm if $f$ is in $L^{2}$. However, in general, for $p$ different from 2 the series fails to converge in the $L^{p}$ norm unless a suitable summation method is applied. Indeed, as proved by Askey and Wainger in [3] the series converges only if $4 / 3<p<4$. Thus we are led to consider the Riesz and Cesàro means of the Hermite expansion.

The Hermite functions are the eigenfunctions of the elliptic operator $\left(-\partial_{x}^{2}+\right.$ $\left.x^{2}\right)$. So in accordance with the general theory of the eigenfunction expansions associated with elliptic operators, we define the Riesz means for the Hermite series as follows. Let $\alpha$ and $R$ be two positive numbers. The Riesz means of order $\alpha$, denoted by $S_{R}(\alpha) f$ is defined by

$$
S_{R}(\alpha) f(x)=\sum_{N \leq R}(1-N / R)^{\alpha} f^{\wedge}(n) \varphi_{n}(x)
$$

where $N=(2 n+1)$. The following formula connecting Riesz means of different order is useful. If $\alpha$ and $\beta$ are positive, then we have the formula

$$
S_{R}(\alpha+\beta)=\frac{\Gamma(\alpha+\beta+1)}{\Gamma(\alpha+1) \Gamma(\beta)} \int_{0}^{1}(1-t)^{\beta-1} S_{R t}(\alpha) d t .
$$

From this equation it follows that the convergence of $S_{R}(\alpha) f$ implies the convergence of $S_{R}(\beta) f$ for any $\beta>\alpha$. To introduce the Cesàro means let us define $A_{k}(\alpha)$ by the formula $A_{k}(\alpha)=\Gamma(k+\alpha+1) /\{\Gamma(k+1) \Gamma(\alpha+1)\}$ for $\alpha$ greater than -1 . The $n$th Cesàro means of order $\alpha$ is defined by

$$
C_{n}(\alpha) f(x)=\left(A_{n}(\alpha)\right)^{-1} \sum_{k \leq n} A_{n-k}(\alpha) f^{\wedge}(k) \varphi_{k}(x) .
$$

There is a formula connecting two Cesàro means of different orders. When $\alpha>-1$ and $\beta>-1$

$$
C_{n}(\alpha+\beta) f(x)=\left(A_{n}(\alpha+\beta)\right)^{-1} \sum_{k \leq n} A_{n-k}(\beta) A_{k}(\alpha) C_{k}(\alpha) f(x) .
$$

Another interesting result is the following theorem of Gergen [10] which expresses the Cesàro means in terms of the Riesz means and vice versa. 
Theorem (Gergen). Let $k$ be the integral part of $\alpha$. There exists two functions $U(x)$ and $V(x), U(x)=O\left(x^{-2}\right)$, as $x \rightarrow \infty, U(x)=O\left(x^{k-\alpha+1}\right)$, as $x \rightarrow 0$; $V(x)=O\left(x^{-2}\right)$, as $x \rightarrow \infty, V(x)=O\left(x^{\alpha}\right)$, as $x \rightarrow 0$ such that we have: $S_{R}(\alpha)=n^{-\alpha} \sum_{k \leq n} V(n-k) A_{k}(\alpha) C_{k}(\alpha)$ and

$$
C_{n}(\alpha)=\left(A_{n}(\alpha)\right)^{-1} \int_{0}^{\infty} U(n+1-t) t^{\alpha} S_{t}(\alpha) d t .
$$

From this theorem it is clear that the convergence of one means implies the convergence of the other. For technical reasons we consider the Riesz means rather than the Cesàro means. Before proceeding to consider the Riesz means let us pause for a moment to see why $1 / 6$ is a possible candidate for the critical index.

Suppose the Cesàro means $C_{n}(\alpha) f$ of an $L^{p}$ function converges to $f$ in the norm. Then $C_{n}(\alpha)$ considered as operators acting on $L^{p}$ would be uniformly bounded. Since the partial sums $S_{n}$ are Cesàro means of order 0 , we can express them in terms of $C_{n}(\alpha)$ as follows [2]:

$$
S_{n} f(x)=\sum_{k \leq n} A_{k}(\alpha) A_{n-k}(-\alpha-1) C_{k}(\alpha) f(x) .
$$

From this relation it follows that

$$
\left|f^{\wedge}(n)\right|\left\|\varphi_{n}\right\|_{p} \leq C \sum_{k \leq n}\left|A_{k}(\alpha) A_{n-k}(-\alpha-1)\right|\|f\|_{p} .
$$

If we use the fact that $A_{k}(\alpha)=O\left(k^{\alpha}\right)$, we obtain immediately $\left|f^{\wedge}(n)\right|\left\|\varphi_{n}\right\|_{p} \leq$ $C N^{\alpha}\|f\|_{p}$. Let $q$ be the exponent conjugate to $p$. By choosing an $f$ such that $\left|\int f(x) \varphi_{n}(x) d x\right| \geq \frac{1}{2}\|f\|_{p}\left\|\varphi_{n}\right\|_{q}$ we get $\left\|\varphi_{n}\right\|_{p}\left\|\varphi_{n}\right\|_{q} \leq C N^{\alpha}$. Now it is easy to get a lower bound for $\left\|\varphi_{n}\right\|_{p}\left\|\varphi_{n}\right\|_{q}$.

Lemma 2.1. There is a constant $C$ such that for large $n$ the following estimates hold:

$$
\left\|\varphi_{n}\right\|_{p} \geq C N^{-1 / 12} N^{-1 / 6 p} ; \quad\left\|\varphi_{n}\right\|_{q} \geq C N^{-1 / 4} N^{1 / 2 q} .
$$

Proof. To prove the first inequality we use the following asymptotic property of $\varphi_{n}(x)$ which is proved in [26]. Let $x=\left(N^{1 / 2}-2^{-1 / 2} 3^{-1 / 3} n^{-1 / 6} t\right)$ where $t$ is bounded and let $\operatorname{Ai}(t)$ denote the Airy function. We have $\varphi_{n}(x)=$ $3^{1 / 3} 2^{1 / 4} \pi^{-1} n^{-1 / 12}\left(\mathrm{Ai}(t)+O\left(n^{-2 / 3}\right)\right)$. We can find an $\varepsilon>0$ such that $|\mathrm{Ai}(t)| \geq c$ for $|t| \leq \varepsilon$ for some constant $c$. Therefore, for $|t| \leq \varepsilon\left|\varphi_{n}(x)\right| \geq c_{1} n^{-1 / 12}-$ $c_{2} n^{-2 / 3}$. From the above by choosing $n$ large we obtain for $N^{1 / 2}-\delta N^{-1 / 6} \leq$ $x \leq N^{1 / 2}+\delta N^{-1 / 6},\left|\varphi_{n}(x)\right| \geq C N^{-1 / 12}$. Integrating over the interval $N^{1 / 2}-$ $\delta N^{-1 / 6} \leq x \leq N^{1 / 2}+\delta N^{-1 / 6}$ we get $\left\|\varphi_{n}\right\|_{p} \geq C N^{-1 / 12} N^{-1 / 6 p}$. This proves the first estimate. To prove the other estimate we use the following result of Muckenhoupt.

Let $w(x)$ be a measurable function and $1 \leq p \leq \infty$. Let $E_{y}$ be the interval $\left[\frac{3}{4} y^{1 / 2}, y^{1 / 2}-1\right]$ and $I_{y}$ be the set of all integers $n$ such that $y \leq 2 n+1 \leq$ 
$y+y^{1 / 3}$. Then there exists $y_{0}$ and $C>0$ depending only on $p$ such that for $y \geq y_{0}$

$$
\left(\int_{E_{y}}\left|\varphi_{n}(x) w(x)\right|^{p} d x\right)^{1 / p} \geq C\left(\int_{E_{y}}\left|w(x) /\left(y-x^{2}\right)^{1 / 4}\right|^{p} d x\right)^{1 / p}
$$

for at least $3 / 5$ of the integers $n$ in $I_{y}$.

Taking $w=1$ in the above result we immediately obtain the following estimate:

$$
\left(\int_{E_{y}}\left|\varphi_{n}(x)\right|^{p} d x\right)^{1 / p} \geq C\left(\int_{E_{y}}\left(y-x^{2}\right)^{-p / 4} d x\right)^{1 / p} \geq C y^{-1 / 4} y^{1 / 2 p}
$$

Since $y$ is comparable with $N$ we obtain $\left\|\varphi_{n}\right\|_{q} \geq c N^{-1 / 4} N^{1 / 2 q}$. Hence the lemma.

Theorem 2.1. If the operators $C_{n}(\alpha)$ are uniformly bounded on $L^{p}$ for a $p \leq 4$ then we necessarily have $p \geq 4 /(6 \alpha+3)$. In particular $C_{n}(\alpha) f$ cannot converge in the norm for all $f$ in $L^{1}$ unless $\alpha$ is greater than $1 / 6$.

Proof. As observed above the uniform boundedness of $C_{n}(\alpha)$ implies that $\left\|\varphi_{n}\right\|_{p}\left\|\varphi_{n}\right\|_{q} \leq 2 C N^{\alpha}$. In view of Lemma 2.1 we have

$$
\left\|\varphi_{n}\right\|_{p}\left\|\varphi_{n}\right\|_{q} \geq C^{\prime} N^{-1 / 2+2 / 3 p}
$$

and therefore, we need to have $N^{-1 / 2+2 / 3 p} \leq C N^{\alpha}$ which will be true for large $N$ only if $\alpha \geq-1 / 2+2 / 3 p$ or $p>4 /(6 \alpha+3)$. For $p=1$ this implies $\alpha>1 / 6$.

\section{RiesZ KeRNel fOR the Hermite SERIES}

The aim of this section is to get a good expression for the kernel of the Riesz means of the Hermite series. Before describing how we go about this, let us briefly indicate the methods employed by the previous authors. The Christoffel-Darboux formula came in handy to study the kernel of the partial sums operators. This formula, together with an ingenious device of Pollard [22] gives a good expression for the kernel of $S_{R}(0)$. Then, using the asymptotic estimates for the Hermite and Laguerre polynomials obtained by Erdelyi and Skovgaard [5], Askey and Wainger obtained good estimates for the kernel of $S_{R}(0)$. The other tools they used were Hardy's inequality and the theory of Hilbert transform.

An idea of Campbell was used by Poiani in the study of the Cesàro means of order 1 of the Hermite and Laguerre series. An exact expression for the Cesàro kernel of the Laguerre series was obtained by Campbell in [4] and was given in terms of a differential operator. This technique was already used by Ernst in 1969 to study the $L^{p}$ convergence of $(C, 1)$ means of Laguerre series. Poiani used this technique together with the asymptotic estimates to get good 
estimates for the Cesàro kernel. To study the Cesàro kernels of fractional order Markett used the product formulas of the Laguerre polynomials as the starting point. He defined certain operators called the Laguerre translation operators and expressed the kernels in terms of these operators. He obtained good estimates when $\alpha>1 / 2$ but his method failed to yield good estimates when $0<\alpha<1 / 2$.

Our investigations of the Hermite series are based on Mehler's formula. For technical reasons we consider the Riesz means instead of the usually considered Cesàro means. In view of the theorem of Gergen both means converge or diverge together. We obtain an expression for the kernel of the Riesz means in terms of certain oscillatory integrals. This point of view had already been taken by E. Kogbetliantz [12] who treated the pointwise convergence of the Laguerre and Hermite series at length around 1935. He used the method of steepest descent to study the kernel. But unfortunately, as pointed out by Poiani in [21], his investigations were based on an erroneous estimate. We use the method of stationary phase to estimate the oscillatory integrals and we do not need any asymptotic estimates of the Hermite polynomials.

Let us start with the Mehler kernel. For $|r|<1$, the Mehler kernel $M_{r}(x, y)$ is defined by

$$
M_{r}(x, y)=\sum_{n \geq 0} r^{n} \varphi_{n}(x) \varphi_{n}(y) .
$$

As proved in [26] this series can be summed to give the following formula:

$$
M_{r}(x, y)=\pi^{-1 / 2}\left(1-r^{2}\right)^{-1 / 2} \exp \left(B_{r}(x, y)\right)
$$

where $B_{r}(x, y)=-\frac{1}{2}\left(x^{2}+y^{2}\right)\left\{\left(1+r^{2}\right) /\left(1-r^{2}\right)\right\}+2 r x y /\left(1-r^{2}\right)$. Let us define a related kernel $G_{r}(t, x, y)$ in the following way. For $0<r<1$, this kernel is defined by the series

$$
G_{r}(t, x, y)=\sum_{n \geq 0} e^{-(2 n+1) i t} r^{n} \varphi_{n}(x) \varphi_{n}(y) .
$$

In terms of the Mehler kernel, $G_{r}(t, x, y)=e^{-i t} M_{\rho}(x, y)$ where $\rho=r e^{-2 i t}$. Let $G(t, x, y)$ be the limit of $G_{r}(t, x, y)$ as $r$ tends to 1 which exists whenever $\sin 2 t$ is different from zero. A simple calculation shows that $G(t, x, y)=$ $c_{0}(\sin 2 t)^{-1 / 2} e^{i \varphi(t)}$ where $c_{0}$ is a constant and $\varphi$ is given by

$$
\varphi(t)=-x y \operatorname{cosec} 2 t+\frac{1}{2}\left(x^{2}+y^{2}\right) \cot 2 t .
$$

We will now prove the following lemma which gives an integral representation of the Riesz kernel in terms of $G(t, x, y)$.

Lemma 3.1. The kernel $S_{R}(x, y)=S_{R}(\alpha ; x, y)$ of the Riesz means $S_{R}(\alpha)$ is given by the integral

$$
S_{R}(x, y)=c R \operatorname{Re}\left\{\int_{0}^{\infty} g(R t) G(t, x, y) d t\right\}
$$


where $g$ is the inverse Fourier transform of the function $h$ defined to be $(1-|t|)^{\alpha}$ when $|t|<1$ and 0 otherwise and $c$ is a constant.

Proof. We start with the following observation. With $N=2 n+1$ and $h$ defined as above the kernel of Riesz means of order $\alpha$ is given by the sum $S_{R}(x, y)=\sum h(N / R) \varphi_{n}(x) \varphi_{n}(y)$. We write this as a limit

$$
S_{R}(x, y)=\operatorname{Lim}_{r \rightarrow 1} \sum h(N / R) r^{n} \varphi_{n}(x) \varphi_{n}(y) .
$$

We will now get an integral expression for the right-hand side. Since the functions $h$ and $g$ are both in $L^{1}$ (we will see shortly why this is so with $g$ ), by Fourier inversion we can write $h(\lambda / R)=R \int g(R t) e^{-i \lambda t} d t$. Now, multiplying (3.3) by $R g(R t)$ on both sides we get

$$
R g(R t) G_{r}(t, x, y)=\sum_{n \geq 0} e^{-(2 n+1) i t} R g(R t) r^{n} \varphi_{n}(x) \varphi_{n}(y) .
$$

Since the Hermite functions are uniformly in $L^{\infty}$ the above series converges absolutely and uniformly in $x$ and $y$. Therefore, term by term integration is possible. Integrating both sides and noting the inversion formula for $h$ we obtain

$$
\int R g(R t) G_{r}(t, x, y) d t=\sum h\left(\frac{N}{R}\right) r^{n} \varphi_{n}(x) \varphi_{n}(y)
$$

Thus $S_{R}(x, y)=\lim _{r \rightarrow 1} \int R g(R t) G_{r}(t, x, y) d t$. We will presently show that we can pass to the limit under the integral sign.

Defining

$$
B_{r}(t)=-\frac{1}{2}\left(x^{2}+y^{2}\right)\left\{\left(1+r^{2} e^{-4 i t}\right) /\left(1-r^{2} e^{-4 i t}\right)\right\}+2 r e^{-2 i t} x y /\left(1-r^{2} e^{-4 i t}\right)
$$

and $A_{r}(t)=\left(e^{2 i t}-r^{2} e^{-2 i t}\right)^{-1 / 2}$ we can write

$$
G_{r}(t, x, y)=\pi^{-1 / 2} A_{r}(t) \exp \left(B_{r}(t)\right) .
$$

We claim that whenever $\sin 2 t$ is different from zero $\left|A_{r}(t)\right| \leq C(\sin 2 t)^{-1 / 2}$ and $\left|\exp B_{r}(t)\right| \leq C$, for $1 / 2 \leq r \leq 1$. An easy calculation shows that $\left|A_{r}(t)\right|^{-4}=$ $\left(1-r^{2}\right)^{2}+4 r^{2} \sin ^{2} 2 t$ from which follows the estimate for $A_{r}(t)$. Another calculation shows that

$$
\begin{aligned}
& \operatorname{Re}\left(1+r^{2} e^{-4 i t}\right) /\left(1-r^{2} e^{-4 i t}\right)=\left|A_{r}(t)\right|^{4}\left(1-r^{4}\right), \\
& \operatorname{Re} r e^{-2 i t} /\left(1-r^{2} e^{-4 i t}\right)=\left|A_{r}(t)\right|^{4} r\left(1-r^{2}\right) \cos 2 t .
\end{aligned}
$$

The claim about $B_{r}(t)$ will follow if we show that

$$
-\frac{1}{2}\left(1-r^{2}\right)\left\{\left(x^{2}+y^{2}\right)\left(1+r^{2}\right)-4 x y r \cos 2 t\right\}<0 \text {. }
$$

When $x y \cos 2 t$ is negative there is nothing to prove. So with $x y \cos 2 t \geq 0$, we consider the function $f(r)=\left(x^{2}+y^{2}\right)\left(1+r^{2}\right)-4 x y r \cos 2 t$. The first derivative vanishes at the point $r=2 x y \cos 2 t\left(x^{2}+y^{2}\right)^{-1}$ where $f(r)$ takes 
the minimum. A calculation shows that the minimum value is $\left(x^{2}+y^{2}\right)-$ $4 x^{2} y^{2} \cos ^{2} 2 t\left(x^{2}+y^{2}\right)^{-1}$ which is clearly positive. Hence the claim.

Therefore, by the dominated convergence theorem we can pass to the limit in (3.5) getting $S_{R}(x, y)=\int R g(R t) G(t, x, y) d t$. Since the function $h$ is real and even $g$ is also real and even and so

$$
S_{R}(x, y)=c R \operatorname{Re}\left\{\int_{0}^{\infty} g(R t) G(t, x, y) d t\right\} .
$$

Thus we get an expression for the kernel in terms of an oscillatory integral. Hence the lemma.

For studying the oscillatory integral appearing in the lemma we need to know how the function $g$ behaves at infinity. An explicit calculation of $g$ is possible. Assume that $0<\alpha<1$. Since $h$ is an even function $g$ is real and is given by

$$
g(t)=\int_{0}^{1}(1-s)^{\alpha} e^{i t s} d s+\int_{0}^{1}(1-s)^{\alpha} e^{-i t s} d s .
$$

By making a change of variable we have

$$
g(t)=e^{-i t} \int_{0 \leq t \leq 1} s^{\alpha} e^{i t s} d s+e^{i t} \int_{0 \leq t \leq 1} s^{\alpha} e^{-i t s} d s .
$$

After an integration by parts we are left with

$$
g(t)=i \alpha t^{-1}\left\{e^{-i t} \int_{0 \leq t \leq 1} s^{\alpha-1} e^{-i t s} d s-e^{i t} \int_{0 \leq t \leq 1} s^{\alpha-1} e^{i t s} d s\right\} .
$$

Consider the first integral. We write this as

$$
e^{-i t} \int_{t>0} s^{\alpha-1} e^{-i t s} d s-e^{-i t} \int_{t>1} s^{\alpha-1} e^{i t s} d s .
$$

For the first part we have the formula (see $[19$, p. 98])

$$
\int_{0}^{\infty} s^{\alpha-1} e^{i t s} d s=\Gamma(\alpha) e^{i \pi \alpha / 2} t^{-\alpha}
$$

One more integration by parts shows that $e^{i t} \int_{t>1} s^{\alpha-1} e^{i t s} d s=O\left(t^{-1}\right)$. The derivative of this last integral also is seen to have the same growth property. Thus, we have proved

Lemma 3.2. Assume that $0<\alpha<1$. Then $g$ is a bounded function and for $t \geq 1$ we have

$$
g(t)=c_{1} t^{-\alpha-1} e^{i t}+c_{2} t^{-\alpha-1} e^{-i t}+c_{3} t^{-2} g_{0}(t)
$$

where $g_{0}(t)$ is bounded together with its derivative. In particular the function $g$ is integrable.

Thus to get an estimate for the Riesz kernel we have to study certain oscillatory integrals. Certain reductions in the expression of the kernel is possible. Since the phase function $\varphi(t)$ is periodic with period $\pi$ it is enough to consider the integrals $R \int_{0 \leq t \leq \pi} g\{R(t+k \pi)\} G(t, x, y) d t$. Among these integrals 
the most difficult one to estimate is the integral corresponding to $k=0$. In fact, we will estimate only this, since the estimation of other integrals are similar.

\section{Estimation OF THE RIESZ KeRNEL}

As indicated in the previous section, we need to estimate

$$
R \int_{0 \leq t \leq \pi} g(R t) G(t, x, y) d t
$$

Further reduction of this integral is possible. By making a change of variable, we can reduce everything to the estimation of the following two integrals:

$$
\begin{gathered}
I=R \int_{0 \leq t \leq \pi / 4} g(R t)(\sin 2 t)^{-1 / 2} e^{i \varphi(t)} d t, \\
J=R \int_{0 \leq t \leq \pi / 4} g\{R(t+k \pi)\}(\sin 2 t)^{-1 / 2} e^{i \varphi^{*}(t)} d t,
\end{gathered}
$$

where $\varphi^{*}(t)=x y \operatorname{cosec} 2 t+\frac{1}{2}\left(x^{2}+y^{2}\right) \cot 2 t$ and $k$ is different from zero. Again we will only estimate $I$. The estimation of $J$ is similar. In fact, it is a lot easier than the estimation of $I$. In what follows $C$ will denote a generic constant which varies from one place to another. Also we are assuming that $1 / 6<\alpha<1 / 2$.

Let $\theta$ be a smooth function which vanishes for $t \leq 1 / 2$ and is identically one for $t \geq 1$. We split $I$ into two parts, viz. $I=A_{0}+A$ where

$$
\begin{gathered}
A=R \int \theta(R t) g(R t)(\sin 2 t)^{-1 / 2} e^{i \varphi(t)} d t, \\
A_{0}=R \int\{1-\theta(R t)\} g(R t)(\sin 2 t)^{-1 / 2} e^{i \varphi(t)} d t,
\end{gathered}
$$

both integrals being extended from 0 to $\pi / 4$. The estimation of $A_{0}$ is easy. A mere integration by parts gives a good estimate.

For that purpose we need an estimate on the first derivative of $\varphi$. Since we have $-\varphi^{\prime}(t) \sin ^{2} 2 t=4 x y \sin ^{2} 2 t+|x-y|^{2}$ we get the estimate

$$
-\varphi^{\prime}(t) \sin ^{2} 2 t \geq \frac{1}{2}|x-y|^{2}, \quad \text { for } 0 \leq t \leq \pi / 4 \text {. }
$$

Another estimate we need is the following one which bounds the second derivative of $\varphi$ in terms of its first derivative. We claim that

$$
\left|\varphi^{\prime \prime}(t) \sin 2 t\right| \leq 4\left|\varphi^{\prime}(t)\right|, \quad \text { for } 0 \leq t \leq \pi / 4 \text {. }
$$

To prove the claim we have $\varphi^{\prime \prime}(t) \sin ^{3} 2 t=4\left\{-4 x y \sin ^{4} t+(x-y)^{2} \cos 2 t\right\}$. Therefore, when $x y \geq 0$ it is clear that $\left|\varphi^{\prime \prime}(t) \sin ^{3} 2 t\right| \leq 4\left|\varphi^{\prime}(t) \sin ^{2} 2 t\right|$. When $x y \leq 0$ we need to check if

$$
-4 x y \sin ^{4} t+(x-y)^{2} \cos 2 t \leq 4 x y \sin ^{2} t+(x-y)^{2} .
$$

If this is not so we will have for some $t_{0},-4 x y \sin ^{4} t_{0}+(x-y)^{2} \cos 2 t_{0}>$ $4 x y \sin ^{2} t_{0}+(x-y)^{2}$ or $-4 x y \sin ^{2} t_{0}\left\{1+\sin ^{2} t_{0}\right\}>2(x-y)^{2} \sin ^{2} t_{0}$ which implies $-2 x y \sin ^{4} t_{0}>x^{2}+y^{2}$. Since this is not true the claim is proved. We can now prove the following proposition. 
Proposition 4.1. There exists a constant $C$ independent of $x, y$ and $R$ such that

$$
\left|A_{0}\right| \leq C R^{1 / 2}\left(1+R^{1 / 2}|x-y|\right)^{-\alpha-5 / 6} .
$$

Proof. Actually we can prove a better estimate. Since $g(t)$ is bounded, we get the obvious estimate $\left|A_{0}\right| \leq C R^{1 / 2}$. Integrating by parts and using estimates (4.5) and (4.6) we obtain $\left|A_{0}\right| \leq C R^{-1 / 2}|x-y|^{-2}$. Combining the two estimates we have $\left|A_{0}\right| \leq C R^{1 / 2}\left(1+R|x-y|^{2}\right)^{-1}$. This proves the proposition since $R^{1 / 2}\left(1+R|x-y|^{2}\right)^{-1}$ is dominated by $R^{1 / 2}\left(1+R^{1 / 2}|x-y|\right)^{-\alpha-5 / 6}$.

To estimate $A$ we use the fact that $g$ contains three terms as given in Lemma 3.2. We estimate only the first two integrals coming from the terms $t^{-\alpha-1} e^{i t}$ and $t^{-\alpha-1} e^{-i t}$. The estimation of the integral involving $t^{-2} g_{0}(t)$ is easy and will not be considered. Thus, we have to estimate the following two oscillatory integrals:

$$
\begin{aligned}
& K=R^{-\alpha} \int \theta(R t) t^{-\alpha-1}(\sin 2 t)^{-1 / 2} e^{i R t} e^{i \varphi(t)} d t, \\
& L=R^{-\alpha} \int \theta(R t) t^{-\alpha-1}(\sin 2 t)^{-1 / 2} e^{-i R t} e^{i \varphi(t)} d t .
\end{aligned}
$$

Our aim is to prove the following proposition which gives a good estimate for the term $A$.

Proposition 4.2. There is a constant $C$ independent of $x, y$ and $R$ such that

$$
|A| \leq C R^{1 / 2}\left(1+R^{1 / 2}|x-y|\right)^{-\alpha-5 / 6} .
$$

For $L$ we can actually prove the estimate $R^{1 / 2}\left(1+R^{1 / 2}|x-y|\right)^{-3 / 2}$. This estimate of $L$ follows from an integration by parts. By letting $\sigma(t)=-R t+\varphi(t)$ we calculate the first derivative of $\sigma$. We have $-\sigma^{\prime}(t) \sin ^{2} 2 t=R \sin ^{2} 2 t+$ $4 x y \sin ^{2} t+(x-y)^{2}$. Since $\sin 2 t \geq c t$ for $0 \leq t \leq \pi / 4$ we get

$$
\left|\sigma^{\prime}(t) \sin ^{2} 2 t\right| \geq C\left\{R t^{2}+(x-y)^{2}\right\} .
$$

Since $\varphi^{\prime \prime}(t)=\sigma^{\prime \prime}(t)$, in view of (4.6), we get another estimate

$$
\left|\sigma^{\prime \prime}(t) \sin 2 t\right|=\left|\varphi^{\prime \prime}(t) \sin 2 t\right| \leq 4\left|\varphi^{\prime}(t)\right| \leq 4\left|\sigma^{\prime}(t)\right| .
$$

Integrating $L$ by parts, the boundary term is seen to be bounded by

$$
R^{1 / 2}\left(1+R^{1 / 2}|x-y|\right)^{-\alpha-5 / 6} .
$$

In view of estimates (4.12) and (4.13), it is easily seen that the differentiated terms are bounded by the integral $R^{-\alpha} \int_{1 / R \leq t \leq \pi / 4}\left(R t^{2}+(x-y)^{2}\right)^{-1} t^{-\alpha-1 / 2} d t$. This last integral is bounded by $R^{-\alpha-1} \int_{1 / R \leq t \leq \pi / 4} t^{-\alpha-5 / 2} d t \leq C R^{1 / 2}$. It is also bounded by

$$
R^{-\alpha-1 / 4}|x-y|^{-3 / 2} \int_{1 / R \leq t \leq \pi / 4} t^{-\alpha-1} d t \leq C R^{-1 / 4}|x-y|^{-3 / 2},
$$


which in turn is bounded by $R^{-\alpha / 2+1 / 12}|x-y|^{-\alpha-5 / 6}$ when $|x-y| \geq R^{-1 / 2}$ as $1 / 6<\alpha<1 / 2$. Combining $R^{1 / 2}$ and $R^{-\alpha / 2+1 / 12}|x-y|^{-\alpha-5 / 6}$ we get $|L| \leq C R^{1 / 2}\left(1+R^{1 / 2}|x-y|\right)^{-\alpha-5 / 6}$.

To study $K$ let us replace $x$ and $y$ by $R^{1 / 2} x$ and $R^{1 / 2} y$ in (4.9) for the sake of convenience and consider the integral

$$
K^{*}=R^{-\alpha} \int \theta(R t) t^{-\alpha-1}(\sin 2 t)^{-1 / 2} e^{i R t} e^{i R \varphi(t)} d t .
$$

Putting $\psi(t)=t+\varphi(t)$, we are looking at integrals of the form

$$
I=\int \omega(t) e^{i R \psi(t)} d t
$$

We are interested in the asymptotic behavior of this integral as $R$ tends to infinity. If the derivative $\psi^{\prime}$ never vanishes on the support of $\omega$, then it follows that $I=O\left(R^{-k}\right)$ for all $k$. The method of stationary phase asserts that the main contribution of the integral comes from the points where the first derivative of $\psi$ is zero. The following lemma of Van der Corput is the main tool which we use to study the oscillatory integral $K^{*}$. The proof of this lemma is given in Stein [24].

Lemma 4.1. Suppose $\psi$ is a real valued smooth function defined on $[a, b]$. Assume that $\left|\psi^{(k)}(t)\right|$ is bounded away from 0 . When $k=1$ we further assume that $\psi^{\prime}(t)$ is monotonic. Then we have

$$
\left|\int_{[a, b]} \omega(t) e^{i R \psi(t)} d t\right| \leq C_{k} R^{-1 / k}\left\{|\omega(b)|+\int_{[a, b]}\left|\omega^{\prime}(t)\right| d t\right\} .
$$

To apply this lemma to our integral we have to treat several cases. When there are no stationary points we apply the lemma with $k=1$ and when there are two distinct stationary points we apply the lemma with $k=2$. We also have to consider the case when the two stationary points are close to each other. In that case we apply the lemma with $k=3$. An easily obtained estimate is $\left|K^{*}\right| \leq C R^{1 / 2}$. This follows by a simple integration. If we combine this estimate with $\left|K^{*}\right| \leq C R^{-\alpha-1 / 3}|x-y|^{-\alpha-5 / 6}$ to be proved for $|x-y| \geq 2 R^{-1}$ we will get the estimate $\left|K^{*}\right| \leq C R^{1 / 2}(1+R|x-y|)^{-\alpha-5 / 6}$ which will at once prove the proposition.

Getting the estimate $\left|K^{*}\right| \leq C R^{-\alpha-1 / 3}|x-y|^{-\alpha-5 / 6}$ is easy when $|x-y|$ is large. We get a lower bound for the third derivative of $\psi$ and apply Lemma 4.1. We claim that $\left|\psi^{\prime \prime \prime}(t)\right| \geq 4(x-y)^{2}$ for $0 \leq t \leq \pi / 4$. Putting $\cos 2 t=\lambda$, $x y=b$ and $x^{2}+y^{2}=a^{2}$ we easily calculate $\psi^{\prime \prime \prime}$. We have that

$$
\left|\psi^{\prime \prime \prime}(t) \sin ^{4} 2 t\right|=8\left(2 a^{2} \lambda^{2}-b \lambda^{3}-5 b \lambda+a^{2}\right) .
$$

To prove the claim it is therefore enough to check if $2\left(2 a^{2} \lambda^{2}-b \lambda^{3}-5 b \lambda+a^{2}\right) \geq$ $\left(a^{2}-2 b\right)$. When $b$ is negative there is nothing to check. When $b$ is positive we will show that $\left(2 a^{2} \lambda^{2}-b \lambda^{3}-5 b \lambda+a^{2}\right) \geq\left(a^{2}-2 b\right)$. Since $a^{2} \geq 2 b$ it is 
enough to check if $G(\lambda)=4 \lambda^{2}-\lambda^{3}-5 \lambda+2 \geq 0$ for $0 \leq \lambda \leq 1$ which is true as $G(\lambda)$ attains a minimum of 0 at $\lambda=1$. This proves the claim. We are now ready to prove the following lemma.

Lemma 4.2. When $|x-y| \geq 2 \delta$, we have the estimate

$$
\left|K^{*}\right| \leq C R^{-\alpha-1 / 3}|x-y|^{-\alpha-5 / 6} \text {. }
$$

Proof. Before applying Lemma 4.1 we have to do an integration by parts:

$$
K^{*}=i R^{-\alpha-1} \int \theta(R t) t^{-\alpha-1}(\sin 2 t)^{3 / 2}\left(4 x y \sin ^{2} t+(x-y)^{2}\right)^{-1} e^{i R t} d\left(e^{i R \varphi(t)}\right) .
$$

The boundary term is bounded by a constant times $R^{-\alpha-1}|x-y|^{-2}$. Since $1 / 6<\alpha<1 / 2$ and $|x-y|$ is bigger than $2 \delta$ this is bounded by $R^{-\alpha-1 / 3}|x-y|^{-\alpha-5 / 6}$. When differentiation falls on the integrand all but one term give the same bound as above. The only one term to be estimated is given by

$$
K^{* *}=R^{-\alpha} \int \theta(R t) t^{-\alpha-1}(\sin 2 t)^{3 / 2}\left(4 x y \sin ^{2} t+(x-y)^{2}\right)^{-1} e^{i R \psi(t)} d t .
$$

Applying Lemma 4.1 to $K^{* *}$ we get

$$
\left|K^{* *}\right| \leq C R^{-\alpha-1 / 3}|x-y|^{-2 / 3-2}\left\{1+\int_{t \geq 1 / R} t^{-\alpha-1 / 2} d t\right\} .
$$

Since $\alpha<1 / 2$ and $|x-y| \geq 2 \delta$ we have the estimate

$$
\left|K^{* *}\right| \leq C R^{-\alpha-1 / 3}|x-y|^{-\alpha-5 / 6} \text {. }
$$

Hence the lemma.

Having estimated $K^{*}$ for $|x-y| \geq 2 \delta$, we now turn our attention to estimate that for the region $2 R^{-1} \leq|x-y| \leq 2 \delta$. By putting $(x-y)^{2}=4 \beta^{2}$ we want to estimate $K^{*}$ for the region $R^{-1} \leq \beta \leq \delta$. We split the integral $K^{*}$ into two parts by writing $K^{*}=B_{0}+B$ where

$$
\begin{gathered}
B_{0}=R^{-\alpha} \int_{0}^{\beta / 2} \theta(R t) t^{-\alpha-1}(\sin 2 t)^{-1 / 2} e^{i R \psi(t)} d t, \\
B=R^{-\alpha} \int_{\beta / 2}^{\pi / 4} \theta(R t) t^{-\alpha-1}(\sin 2 t)^{-1 / 2} e^{i R \psi(t)} d t .
\end{gathered}
$$

Estimation of the first integral is easy since the first derivative of $\psi$ is bounded away from 0 in the interval of integration. Indeed, since $0 \leq t \leq \beta / 2$ for the first integral and the first derivative of $\psi$ is given by $-\psi^{\prime}(t) \sin ^{2} 2 t=-\sin ^{2} 2 t+$ $4 x y \sin ^{2} t+(x-y)^{2}$ we have $\left|-\psi^{\prime}(t) \sin ^{2} 2 t\right| \geq \beta^{2}$. We also have the estimate $\left|\psi^{\prime \prime}(t)\right|=\left|\varphi^{\prime \prime}(t)\right| \leq 4\left|\varphi^{\prime}(t)\right| \operatorname{cosec} 2 t \leq 4 \operatorname{cosec} 2 t+4\left|\psi^{\prime}(t)\right| \operatorname{cosec} 2 t$. Integrating by parts and using the above estimates, we get the bound $R^{-\alpha-1 / 3}|x-y|^{-\alpha-5 / 6}$ for $B_{0}$. 
Next we consider the term $B$ for the region $R^{-1} \leq \beta \leq \delta$. Now, we have to determine the stationary points of the phase function $\psi$. Putting $\cos 2 t=$ $\lambda$, we see that the first derivative of $\psi$ satisfies the equation $-\psi^{\prime}(t) \sin ^{2} 2 t=$ $\left(\lambda^{2}-2 b \lambda+a^{2}-1\right)$. First consider the case when $b<0$. Since the function $f(\lambda)=\left(\lambda^{2}-2 b \lambda+a^{2}-1\right)$ is increasing it vanishes only if $a^{2} \leq 1$. If we choose $\delta$ to be smaller than $1 / 4$, then for the region $|x-y| \leq 2 \delta$, we have $a^{2} \leq|x-y|^{2} \leq 1 / 4$ and hence there is only one stationary point. This stationary point is given by $\cos 2 t_{1}=b+m$ where $m^{2}=\left(1-x^{2}\right)\left(1-y^{2}\right)$. Observe that $m^{2} \geq 1 / 4$ as $a^{2} \leq 1 / 2$.

When $b \geq 0$, the function $f(\lambda)=\left(\lambda^{2}-2 b \lambda+a^{2}-1\right)$ decreases as long as $0<\lambda<b$, reaches a minimum at $b$ and then increases. When $a^{2} \geq 2 f$ does not vanish at all. There are two neighbouring stationary points $t_{1}$ and $t_{2}$ when $1 / 2 \leq a^{2} \leq 2$ and $b \leq 1$. These are given by the equations $\cos 2 t_{1}=b+m$ and $\cos 2 t_{2}=b-m$. Observe that these two stationary points coincide when either $x=1$ or $y=1$. Finally, when $a^{2} \leq 1 / 2$, there is only one stationary point, namely $t_{1}$. Thus, we have to treat several cases in order to estimate the integral $B$.

Let us start with the case when there is only one stationary point. This comprises the cases when $b<0$ and $b \geq 0$ but $a^{2} \leq 1 / 2$. We need to calculate the second derivative of $\psi$ at the stationary point. A simple calculation shows that

$$
\psi^{\prime \prime}(t)=4 \operatorname{cosec}^{3} 2 t\left\{a^{2} \cos 2 t-b \cos ^{2} 2 t-b\right\}
$$

and $\psi^{\prime \prime}\left(t_{1}\right)=4 m \operatorname{cosec} 2 t_{1}$. Since $m \geq 1 / 2$, we get the lower bound $\psi^{\prime \prime}\left(t_{1}\right) \geq$ $2 \operatorname{cosec} 2 t_{1}$. Having made all these preliminary observations, we can now prove the following lemma.

Lemma 4.3. Assume that $b<0$ or $b \geq 0$ and $a^{2} \leq 1 / 2$. Then for the region $|x-y| \leq 2 \delta$ we have the estimate $|B| \leq C R^{-\alpha-1 / 3}|x-y|^{-\alpha-5 / 6}$ provided $\delta$ is smaller than $1 / 4$.

Proof. First we consider the case when $b$ is negative. In that case it is easily seen that $\psi^{\prime \prime}(t)$ is a decreasing function of $t$ in the interval $\beta / 2 \leq t \leq t_{1}$. This implies, for $\beta / 2 \leq t \leq t_{1}$, the lower bound $\left|\psi^{\prime \prime}(t)\right| \geq \psi^{\prime \prime}\left(t_{1}\right) \geq 2 \operatorname{cosec} 2 t_{1}$. If we apply Lemma 4.1 to the integral

$$
R^{-\alpha} \int_{\beta / 2}^{t_{1}} \theta(R t) t^{-\alpha-1}(\sin 2 t)^{-1 / 2} e^{i R \psi(t)} d t
$$

we get the estimate $C R^{-\alpha-1 / 2}\left\{t_{1}^{-\alpha-1}+\beta^{-\alpha-3 / 2}\left(\sin 2 t_{1}\right)^{1 / 2}\right\}$. Now, another calculation shows that $\sin ^{2} 2 t_{1}=a^{2}-2 b \cos 2 t_{1}$ so that $\frac{1}{2}|x-y|^{2} \leq a^{2} \leq \sin ^{2} 2 t_{1} \leq$ $|x-y|^{2}$. In view of this, we get the estimate $C R^{-\alpha-1 / 2}|x-y|^{-\alpha-1}$ which is bounded by $C R^{-\alpha-1 / 3}|x-y|^{-\alpha-5 / 6}$. 
It remains to consider the integral

$$
R^{-\alpha} \int_{t_{1}}^{\pi / 4} \theta(R t) t^{-\alpha-1}(\sin 2 t)^{-1 / 2} e^{i R \psi(t)} d t
$$

Let $\varepsilon=\frac{1}{20} \sin 2 t_{1}$. We claim that we have the following estimates:

$$
\begin{gathered}
\psi^{\prime \prime}(t) \geq \frac{1}{2} \psi^{\prime \prime}\left(t_{1}\right), \quad \text { for } t_{1} \leq t \leq t_{1}+\varepsilon, \\
\psi^{\prime}(t) \geq 1 / 10, \quad \text { for } t_{1}+\varepsilon \leq t \leq \pi / 4 .
\end{gathered}
$$

Postponing the proof of the claim for a moment let us see how we complete the proof of the lemma in the present case. We split the integral into two parts, one from $t_{1}$ to $t_{1}+\varepsilon$ and the other from $t_{1}+\varepsilon$ to $\pi / 4$. Applying Lemma 4.1 with $k=2$ and $k=1$ and using estimates (4.19) and (4.20) we get the estimates we wanted. Let us return to the proof of the claim now.

If $\varepsilon$ is small enough it is clear that in an $\varepsilon$ neighborhood of $t_{1}$ we can have $\psi^{\prime \prime}(t) \geq \frac{1}{2} \psi^{\prime \prime}\left(t_{1}\right)$ and outside the $\varepsilon$ neighborhood $\psi^{\prime}(t)$ could be bounded away from zero. Our task is to find a suitable $\varepsilon$. To do that we use Taylor's theorem with the integral form of remainder. Since $\psi^{\prime}\left(t_{1}\right)=0$ Taylor's theorem applied to the function $\psi^{\prime}(t)$ gives

$$
\psi^{\prime}(t)=\left(t-t_{1}\right) \psi^{\prime \prime}\left(t_{1}\right)+\int_{t_{1}}^{t}(t-s) \psi^{\prime \prime \prime}(s) d s=\left(t-t_{1}\right) \psi^{\prime \prime}(t)+\int_{t_{1}}^{t}\left(s-t_{1}\right)\left(-\psi^{\prime \prime \prime}(s)\right) d s .
$$

Since

$$
\begin{aligned}
\psi^{\prime \prime \prime}(s)= & -24 \operatorname{cosec}^{3} 2 s \cot 2 s\left\{a^{2} \cos 2 s-b \cos ^{2} 2 s-b\right\} \\
& -8 \operatorname{cosec}^{2} 2 s\left\{a^{2}-2 b \cos 2 t\right\}
\end{aligned}
$$

is negative

$$
\psi^{\prime}(t) \geq\left(t-t_{1}\right) \psi^{\prime \prime}(t), \quad \text { for } t_{1} \leq t \leq \pi / 4 \text {. }
$$

Also $\left|\psi^{\prime \prime \prime}(t)\right|=6 \cot 2 t \psi^{\prime \prime}(t)+8 \operatorname{cosec}^{2} 2 t\left\{a^{2}-2 b \cos 2 t\right\}$ is decreasing for $t_{1} \leq$ $t \leq \pi / 4$ and so we have $\left|\psi^{\prime \prime}(t)-\psi^{\prime \prime}\left(t_{1}\right)\right| \leq\left|\psi^{\prime \prime \prime}\left(t_{1}\right)\right|\left(t-t_{1}\right)$. Since $\psi^{\prime \prime}\left(t_{1}\right)=$ $4 m \operatorname{cosec} 2 t_{1} \geq 2 \operatorname{cosec} 2 t_{1}$ we see that $\left|\psi^{\prime \prime \prime}\left(t_{1}\right)\right| \leq 10 \operatorname{cosec} 2 t_{1} \psi^{\prime \prime}\left(t_{1}\right)$ so that $\left|\psi^{\prime \prime}(t)-\psi^{\prime \prime}\left(t_{1}\right)\right| \leq 10 \operatorname{cosec} 2 t_{1} \psi^{\prime \prime}\left(t_{1}\right)\left(t-t_{1}\right)$. Now if we take $\varepsilon=\frac{1}{20} \sin 2 t_{1}$ then for $t_{1} \leq t \leq t_{1}+\varepsilon$ we get

$$
\psi^{\prime \prime}\left(t_{1}\right)-\psi^{\prime \prime}(t) \leq \frac{1}{2} \psi^{\prime \prime}\left(t_{1}\right) \text { or } \quad \psi^{\prime \prime}(t) \geq \frac{1}{2} \psi^{\prime \prime}\left(t_{1}\right) .
$$

Hence from (4.21), for $t_{1} \leq t \leq t_{1}+\varepsilon$, we get the lower bound $\psi^{\prime}(t) \geq$ $\frac{1}{2}\left(t-t_{1}\right) \psi^{\prime \prime}\left(t_{1}\right)$. From this, since $\psi^{\prime}(t)$ is increasing in the interval $t_{1}+\varepsilon \leq$ $t \leq \pi / 4$, we have $\psi^{\prime}(t) \geq \psi^{\prime}\left(t_{1}+\varepsilon\right) \geq \frac{1}{2} \varepsilon \psi^{\prime \prime}\left(t_{1}\right)$ which proves the claim by the choice of $\varepsilon$.

This takes care of the case when $x y<0$. Next consider the case when $x y \geq 0$. In this case the second derivative of $\psi$ vanishes at the point $t_{0}$ defined by $\cos 2 t_{0}=x / y$ (resp. $\left.y / x\right)$ when $x<y$ (resp. $\left.y<x\right)$. As before 
the stationary point is at $t_{1}$ where $\cos 2 t_{1}=b+m$ and $m \geq 1 / 2$ as $a^{2} \leq 1 / 2$. There are three integrals to consider. First we estimate

$$
R^{-\alpha} \int_{t_{0}}^{\pi / 4} \theta(R t) t^{-\alpha-1}(\sin 2 t)^{-1 / 2} e^{i R \psi(t)} d t .
$$

From the expression $-\psi^{\prime \prime \prime}(t) \sin ^{4} 2 t=8\left(2 a^{2} \lambda^{2}-b \lambda^{3}-5 b \lambda+a^{2}\right)$ it is easily seen that $\psi^{\prime \prime \prime}(t)$ is negative. Hence $\psi^{\prime}(t)$ attains a maximum at $t_{0}$ and then decreases in the interval $t_{1} \leq t \leq \pi / 4$.

We therefore have the estimate $\left|\psi^{\prime}(t)\right| \geq\left|\psi^{\prime}(\pi / 4) \sin ^{2} \pi / 2\right|=\left(1-a^{2}\right) \geq$ $1 / 2$. Applying Lemma 4.1 with $k=1$ we immediately get the estimate. The estimation of the other two integrals is similar to the previous case, namely the case when $x y$ is negative. For example, consider the integral

$$
R^{-\alpha} \int_{\beta / 2}^{t_{1}} \theta(R t) t^{-\alpha-1}(\sin 2 t)^{-1 / 2} e^{i R \psi(t)} d t .
$$

Applying Taylor's theorem to $\psi^{\prime}(t)$ we can show that $\left|\psi^{\prime}(t)\right| \geq\left(t_{1}-t\right) \psi^{\prime \prime}\left(t_{1}\right)$ for $\beta / 2 \leq t \leq t_{1}$. If we set $\varepsilon=\frac{1}{4} \sin 2 t_{1}$ so that $\varepsilon \leq \frac{1}{2} t_{1}$ then for $t_{1}-t \geq \varepsilon$, i.e. for $t \leq t_{1}-\varepsilon,\left|\psi^{\prime}(t)\right| \geq 2 \varepsilon \operatorname{cosec} 2 t_{1} \geq \frac{1}{2}$. Also since $\psi^{\prime \prime}(t)$ is decreasing in the interval $\beta / 2 \leq t \leq t_{1},\left|\psi^{\prime \prime}(t)\right| \geq \psi^{\prime \prime}\left(t_{1}\right) \geq 2 \operatorname{cosec} 2 t_{1}$. Split the integral into two parts and apply Lemma 4.1 as before. Since $\sin ^{2} 2 t_{1}=a^{2}-2 b \cos 2 t_{1} \geq a^{2}-2 b$ we get the estimate $C R^{-\alpha-1 / 3}|x-y|^{-\alpha-5 / 6}$. Finally, the estimation of the integral

$$
R^{-\alpha} \int_{t_{1}}^{t_{0}} \theta(R t) t^{-\alpha-1}(\sin 2 t)^{-1 / 2} e^{i R \psi(t)} d t
$$

is similarly done. Proceeding as above, we can show that the choice $\varepsilon=$ $\frac{1}{20} \sin 2 t_{1}$ works. Thus we have taken care of the case with one stationary point.

Next we consider the case $x^{2}+y^{2} \geq 1 / 2$. In this case there are two stationary points for the function $\psi$ and when either $x=1$ or $y=1$ they coincide with one another. Somehow, the method of stationary phase applied as such to the integral $B$ fails to give a good estimate. So, a more careful analysis of the integral is needed. Let $\delta$ be chosen so small that when $|x-y| \leq 2 \delta$, we have $\frac{1}{2} \beta \leq \frac{1}{3} \beta^{3 / 5}$ and $x y \geq \tau$ for some $\tau$. We split the integral $B$ into two parts, viz. $B=F_{0}+F$ with

$$
\begin{aligned}
F_{0} & =R^{-\alpha} \int_{\rho \beta^{3 / 5}}^{\pi / 4} \theta(R t) t^{-\alpha-1}(\sin 2 t)^{-1 / 2} e^{i R \psi(t)} d t, \\
F & =R^{-\alpha-1} \int_{\beta / 2}^{\rho \beta^{3 / 5}} \theta(R t) t^{-\alpha-1}(\sin 2 t)^{-1 / 2} e^{i R \psi(t)} d t .
\end{aligned}
$$

Here we take $\rho=1$ or $1 / 3$. An application of Lemma 4.1 will give a good estimate for $F_{0}$. To estimate $F$ we first rewrite the phase function in a different form and then apply the method of stationary phase. The next lemma gives the required estimate for $F_{0}$. Recall that we are considering the case $x y \geq 0$ and $x^{2}+y^{2} \geq 1 / 2$. 
Lemma 4.4. For the region $2 R^{-1} \leq|x-y| \leq 2 \delta$, where $\delta$ is small, we have the estimate

$$
\left|F_{0}\right| \leq C R^{-\alpha-1 / 3}|x-y|^{-\alpha-5 / 6} \text {. }
$$

Proof. We are going to apply Lemma 4.1 with $k=3$. So, we need a lower bound for the third derivative of $\psi$. With $\cos 2 t=\lambda$ we have the following expression for the third derivative:

$$
\left|\psi^{\prime \prime \prime}(t)\right|=8\left(2 a^{2} \lambda^{2}-b \lambda^{3}-5 b \lambda+a^{2}\right)\left(1-\lambda^{2}\right)^{-2} .
$$

We claim that for $0 \leq t \leq \pi / 4,\left|\psi^{\prime \prime \prime}(t)\right| \geq x y$. To prove the claim, since $a^{2} \geq 2 b$, it is enough to check if $8\left(4 \lambda^{2}-\lambda^{3}-5 \lambda+2\right) \geq\left(1-\lambda^{2}\right)^{2}$. But $\left(4 \lambda^{2}-\lambda^{3}-5 \lambda+2\right)=(2-\lambda)(1-\lambda)^{2}$ so that we have to check if $8(2-\lambda) \geq(1+\lambda)^{2}$ which is clearly true for $0 \leq \lambda \leq 1$. Thus, $\left|\psi^{\prime \prime \prime}(t)\right| \geq x y \geq \tau$ for $0 \leq t \leq \pi / 4$. Applying Lemma 4.1 with $k=3$ we immediately obtain the following estimate:

$$
\left|F_{0}\right| \leq C R^{-\alpha-1 / 3}|x-y|^{-3 \alpha / 5-9 / 10} .
$$

Since $|x-y| \leq 2 \delta$ and $1 / 6<\alpha<1 / 2$, this gives the estimate $\left|F_{0}\right| \leq$ $C R^{-\alpha-1 / 3}|x-y|^{-\alpha-5 / 6}$.

To estimate $F$ we have to consider two cases. First assume that $1 / 2 \leq$ $x^{2}+y^{2} \leq 4$. We rewrite the function $\psi$ as follows. As $\psi(t)=t-b \operatorname{cosec} 2 t+$ $\frac{1}{2} a^{2} \cot 2 t$ we have

$$
\psi(t)=t+\frac{1}{2}(x-y)^{2} \operatorname{cosec} 2 t-\frac{1}{2}\left(x^{2}+y^{2}\right) \tan t .
$$

The functions $\tan t$ and $\operatorname{cosec} 2 t$ can be expanded in powers of $t$. We then have

$$
\tan t=t+b(t)
$$

$$
\operatorname{cosec} 2 t=\frac{1}{2} t^{-1}+\frac{1}{3} t+a(t)
$$

where $a(t)=O\left(t^{3}\right)$ and $b(t)=O\left(t^{3}\right)$ for $0 \leq t \leq \pi / 4$. Then we have $\psi(t)=$ $\psi_{1}(t)+\psi_{2}(t)$ with

$$
\begin{gathered}
\psi_{1}(t)=\left\{1+\frac{1}{6}(x-y)^{2}-\frac{1}{2}\left(x^{2}+y^{2}\right)\right\} t+\frac{1}{4} t^{-1}(x-y)^{2}, \\
\psi_{2}(t)=\frac{1}{2}(x-y)^{2} a(t)-\frac{1}{2}\left(x^{2}+y^{2}\right) b(t) .
\end{gathered}
$$

Since by definition $(x-y)^{2}=4 \beta^{2}$, we have $\psi_{1}(t)=\left(1-\frac{1}{2} a^{2}\right) t+\beta^{2} t^{-1}+\frac{2}{3} \beta^{2} t$. Also, if we put $w(t)=\theta(R t)(2 t / \sin 2 t)^{1 / 2}$, then we have $|w(t)| \leq C$ for $|t| \leq$ $\pi / 4$. Having made all these preliminary simplifications we consider the integral $F=R^{-\alpha} \int_{\beta / 2}^{\rho \beta^{3 / 5}} w(t) t^{-\alpha-3 / 2} \exp i R\left\{\left(1-\frac{1}{2} a^{2}\right) t+\beta^{2} t^{-1}+\frac{2}{3} \beta^{2} t\right\} \exp \left\{i R \psi_{2}(t)\right\} d t$. 
Lemma 4.5. Assume that $x y \geq 0,1 / 2 \leq x^{2}+y^{2} \leq 4$, and $2 R^{-1} \leq|x-y| \leq 2 \delta$ where $\delta$ is small. Then

$$
|F| \leq C R^{-\alpha-1 / 3}|x-y|^{-\alpha-5 / 6} \text {. }
$$

Proof. First assume that $\lambda^{2}=\left(1-\frac{1}{2} a^{2}+\frac{2}{3} \beta^{2}\right)$ is positive. We make a change of variable in the integral $F$. Changing $t$ into $R \beta^{2} t$ we see that the integral becomes $R^{-2 \alpha-1 / 2} \beta^{-2 \alpha-1} I$ where the integral $I$ is given by

$$
I=\int_{E} t^{-\alpha-3 / 2} w\left(R \beta^{2} t\right) \exp \left\{i\left(R^{2} \beta^{2} \lambda^{2} t+t^{-1}\right)\right\} \exp \left\{i R \psi_{2}\left(R \beta^{2} t\right)\right\} d t
$$

where $E$ is the interval $\frac{1}{2} R^{-1} \beta^{-1} \leq t \leq R^{-1} \beta^{-7 / 5}$. Since the above integral taken from 1 to infinity is bounded we can assume without loss of generality that $R^{-1} \beta^{-7 / 5} \leq 1$.

We have to consider two cases. First assume that $\lambda \geq 2 \beta^{2 / 5}$. Putting $\psi_{1}(t)=$ $R^{2} \beta^{2} \lambda^{2} t+t^{-1}$ the stationary point of $\psi_{1}$ is given by $t_{0}=(R \beta \lambda)^{-1}$. Since $\lambda^{2} \geq 4 \beta^{4 / 5}$, we see that $2 t_{0} \leq R^{-1} \beta^{-7 / 5}$. We write $I$ as the sum of the following three integrals:

$$
\begin{aligned}
& I_{1}=\int_{L} t^{-\alpha-3 / 2} w\left(R \beta^{2} t\right) \exp \left\{i\left(R^{2} \beta^{2} \lambda^{2} t+t^{-1}\right)\right\} \exp \left\{i R \psi_{2}\left(R \beta^{2} t\right)\right\} d t \\
& I_{2}=\int_{M} t^{-\alpha-3 / 2} w\left(R \beta^{2} t\right) \exp \left\{i\left(R^{2} \beta^{2} \lambda^{2} t+t^{-1}\right)\right\} \exp \left\{i R \psi_{2}\left(R \beta^{2} t\right)\right\} d t \\
& I_{3}=\int_{N} t^{-\alpha-3 / 2} w\left(R \beta^{2} t\right) \exp \left\{i\left(R^{2} \beta^{2} \lambda^{2} t+t^{-1}\right)\right\} \exp \left\{i R \psi_{2}\left(R \beta^{2} t\right)\right\} d t
\end{aligned}
$$

where $L, M$ and $N$ are the intervals $\frac{1}{2} R^{-1} \beta^{-1} \leq t \leq \frac{1}{2} t_{0}, \frac{1}{2} t_{0} \leq t \leq 2 t_{0}$, and $2 t_{0} \leq t \leq R^{-1} \beta^{-7 / 5}$, respectively. By setting $\psi(t)=R^{2} \beta^{2} \lambda^{2} t+t^{-1}+R \psi_{2}\left(R \beta^{2} t\right)$ we want to show that the integral

$$
I_{1}=\int_{L} t^{-\alpha-3 / 2} w\left(R \beta^{2} t\right) e^{i \psi(t)} d t
$$

is bounded independent of $x, y$ and $R$. This is done by applying the method of stationary phase. For that purpose we need to get some bounds for the first and second derivatives of $\psi$.

A simple calculation shows that $-\psi^{\prime}(t) t^{2}=1-R^{2} \beta^{2} \lambda^{2} t^{2}-R^{2} \beta^{2} t^{2} \psi_{2}^{\prime}\left(R \beta^{2} t\right)$. Since $t \leq \frac{1}{2} t_{0}$ and $t_{0}=\frac{1}{2}(R \beta \lambda)^{-1}$ we have $R^{2} \beta^{2} \lambda^{2} t^{2} \leq 1 / 4$ for $t$ in $L$. Also since $\psi_{2}^{\prime}(t)=O\left(t^{2}\right)$, we see that $R^{2} \beta^{2} t^{2}\left|\psi_{2}^{\prime}\left(R \beta^{2} t\right)\right| \leq C \beta^{2} \lambda^{-4}$ and as $\lambda \geq 2 \beta^{2 / 5}$ we have $R^{2} \beta^{2} t^{2}\left|\psi_{2}^{\prime}\left(R \beta^{2} t\right)\right| \leq C \beta^{2 / 5}$ which is less than $1 / 2$ for $|x-y| \leq 2 \delta$ provided $\delta$ is small enough. Thus, we have the estimate $-\psi^{\prime}(t) t^{2} \geq 1 / 4$ for $t$ in $L$. We need one more bound for the second derivative of $\psi$. Another calculation shows that $t^{3} \psi^{\prime \prime}(t)=2+R^{3} \beta^{4} t^{3} \psi^{\prime \prime}\left(R \beta^{2} t\right)$. Since $\psi^{\prime \prime}(t)=O(t)$ the above gives $\left|t^{3} \psi^{\prime \prime}(t)\right| \leq 2+C \beta^{2 / 5} \leq C$ for $t$ in $L$. Also we have $w^{\prime}(t)=O(t)$ since $t \leq R^{-1}$ on the support of $\theta^{\prime}(R t)$. 
Having made all these observations we integrate $I_{1}$ by parts. We have

$$
I_{1}=-i \int_{L} t^{-\alpha+1 / 2} w\left(R \beta^{2} t\right)\left(t^{2} \psi^{\prime}(t)\right)^{-1} d\left\{e^{i \psi(t)}\right\}
$$

Since $\alpha<1 / 2$ and $R^{-1} \beta^{-7 / 5} \leq 1$, in view of the above bounds we see that $\left|I_{1}\right| \leq C$ as desired. The estimation of the integral

$$
I_{3}=\int_{N} t^{-\alpha-3 / 2} w\left(R \beta^{2} t\right) e^{i \psi(t)} d t
$$

is similar. To estimate the remaining integral $I_{2}$ we are going to apply Lemma 4.1.

Let us get a bound for $\psi^{\prime \prime}$ when $t$ is in $M$. We have $\psi^{\prime \prime}(t)=2 t^{-3}+$ $R^{3} \beta^{4} \psi^{\prime \prime}\left(R \beta^{2} t\right)$ which gives for $\frac{1}{2} t_{0} \leq t \leq 2 t_{0}$ the lower bound $\left|\psi^{\prime \prime}(t)\right| \geq$ $c\left(t_{0}\right)^{-3}$. Applying Lemma 4.1 and using the fact that $w^{\prime}(t)=O(t)$ we immediately obtain the estimate $\left|I_{2}\right| \leq C(R \beta \lambda)^{\alpha}$. Therefore, we get

$$
R^{-2 \alpha-1 / 2} \beta^{-2 \alpha-1}\left|I_{2}\right| \leq C R^{-\alpha-1 / 2} \beta^{-\alpha-1} .
$$

This takes care of the lemma when $\lambda \geq 2 \beta^{2 / 5}$.

Next we consider the same integral $I$ in the case $\lambda<2 \beta^{2 / 5}$ but now the integral is taken over the interval $E$ defined by $\frac{1}{2} R^{-1} \beta^{-1} \leq t \leq \frac{1}{3} R^{-1} \beta^{-7.5}$. Since now $R^{-1} \beta^{-1} \lambda^{-1} \geq \frac{1}{2} R^{-1} \beta^{-7 / 5}$ there is no stationary point for the function $\psi_{1}(t)=\lambda^{2} t+\beta^{2} t^{-1}$. Calculating the first derivative of $\psi(t)$ we see that $-\psi^{\prime}(t) t^{2}=1-R^{2} \beta^{2} \lambda^{2} t^{2}-R^{2} \beta^{2} t^{2} \psi_{2}^{\prime}\left(R \beta^{2} t\right)$. Since $\lambda<2 \beta^{2 / 5}$ and $t \leq$ $\frac{1}{3} R^{-1} \beta^{-7 / 5}$ we have $R^{2} \beta^{2} \lambda^{2} t^{2} \leq \frac{4}{9} R^{2} \beta^{2} \beta^{4 / 5} R^{-2} \beta^{-14 / 5} \leq 4 / 9$. Also if $\delta$ is chosen sufficiently small we can make $\left|R^{2} \beta^{2} t^{2} \psi_{2}^{\prime}\left(R \beta^{2} t\right)\right| \leq 2 / 9$. Thus, $\left|-\psi^{\prime}(t) t^{2}\right| \geq 1 / 3$ for $t$ in $E$. As in the previous case we can also get bounds for the second derivative of $\psi$. Then an integration by parts gives the required estimate.

Thus we have estimated $F$ under the assumption that $\lambda^{2}=\left(1-\frac{1}{2} a^{2}+\frac{2}{3} \beta^{2}\right)$ is positive. Next assume that $-\lambda^{2}=\left(1-\frac{1}{2} a^{2}+\frac{2}{3} \beta^{2}\right)$ is negative. As before by changing the variable we consider $R^{-2 \alpha-1 / 2} \beta^{-2(x-1} I$ where

$$
I=\int_{E} t^{-\alpha-3 / 2} w\left(R \beta^{2} t\right) \exp \left\{i\left(-R \beta^{2} \lambda^{2} t+t^{-1}\right)\right\} \exp \left\{i R \psi_{2}\left(R \beta^{2} t\right)\right\} d t .
$$

We want to show that the integral is bounded independent of $x, y$ and $R$. We can easily see that for $t$ in $E,\left|\psi^{\prime}(t) t^{2}\right| \geq 1 / 3$ where $\psi(t)=-R \beta^{2} \lambda^{2} t+t^{-1}+$ $R \psi_{2}\left(R \beta^{2} t\right)$. Also we can obtain bounds for the second derivative. Integration by parts gives the required estimate as before.

It remains to consider the case $x^{2}+y^{2}>4$. But this case is very easy. We look at the first derivative of $\psi$. Let $t_{0}$ be the point where $\psi^{\prime \prime}(t)$ vanishes. Since $\psi^{\prime \prime \prime}$ is negative $\psi^{\prime}(t)$ attains maximum at $t_{0}$. A calculation shows that $\psi^{\prime}(t)=\left(1-x^{2}\right)$ when $\cos 2 t_{0}=y / x$ and $\psi^{\prime}(t)=\left(1-y^{2}\right)$ when $\cos 2 t_{0}=x / y$. 
Therefore, as $x^{2}+y^{2}>4,\left|\psi^{\prime}(t)\right| \geq 1$. An integration by parts gives the estimate $|F| \leq C R^{-\alpha-1 / 3}|x-y|^{-\alpha-5 / 6}$.

Now it is time to put all the loose ends together. Combining Propositions 4.1 and 4.2, we have a good estimate for the integral $I$ mentioned in the beginning of this section. In estimating the term $A$ we omitted the contribution from the part $t^{-2} g_{0}(t)$ of $g(t)$. But the estimation of this contribution is easy and it gives a similar estimate. The final estimate of the kernel is stated in the following theorem.

Theorem 4.1. Assume that $1 / 6<\alpha<1 / 2$. Then with a $C$ independent of $x, y$ and $R$ we have

$$
\left|S_{R}(x, y)\right| \leq C R^{1 / 2}\left(1+R^{1 / 2}|x-y|\right)^{-\alpha-5 / 6}+R^{1 / 2}\left(1+R^{1 / 2}|x+y|\right)^{-\alpha-5 / 6}
$$

In the above estimate the contribution $R^{1 / 2}\left(1+R^{1 / 2}|x+y|\right)^{-\alpha-5 / 6}$ comes from the integral $J$ mentioned in the beginning. Note that $J$ is defined in terms of $\varphi^{*}$. Since $\varphi^{*}$ is obtained from $\varphi$ by replacing $y$ by $-y$, the estimation of $J$ does not pose any new problem. If we replace $y$ by $-y$ in the estimate obtained for $I$, we get the estimate for $J . R \int g\{R(t+k \pi)\} G(t, x, y) d t$ the integrals mentioned before are easy to estimate. Each of them gives an estimate $k^{-\alpha-1}$ times $R^{1 / 2}\left(1+R^{1 / 2}|x-y|\right)^{-\alpha-5 / 6}+R^{1 / 2}\left(1+R^{1 / 2}|x+y|\right)^{-\alpha-5 / 6}$ and so we can sum the series to get the final estimate.

\section{SUMmability ReSUlts FOR THE HeRmite EXPANSIONS}

In this section we prove all the results mentioned in the introduction concerning the summability of the Hermite series. All the theorems of this section are easy consequences of the main estimate proved in the last section. Recall that we have

$$
\left|S_{R}(x, y)\right| \leq C\left\{E_{R}(x-y)+E_{R}(x+y)\right\}
$$

where $E_{R}(x)=R^{1 / 2}\left(1+R^{1 / 2}|x|\right)^{-\alpha-5 / 6}$. The Riesz means $S_{R}(\alpha) f$ of an $L^{p}$ function $f$ are given by $S_{R}(\alpha) f(x)=\int S_{R}(x, y) f(y) d y$. Let us denote by $f^{*}$ the function defined by $f^{*}(x)=f(-x)$ and by $E_{R} f(x)$ the convolution of $f$ with $E_{R}(x)$. Then we have

$$
\left|S_{R}(\alpha) f(x)\right| \leq C\left\{E_{R}|f|(x)+E_{R}\left|f^{*}\right|(x)\right\} .
$$

We are now ready to prove the following theorem. In what follows we assume that $f$ is nonnegative without losing any generality.

Theorem 5.1. Assume that $\alpha>1 / 6$ and $1 \leq p \leq \infty$. The Riesz means of order $\alpha$ are uniformly bounded on $L^{p}$, i.e. there is a constant $C$ independent of $f$ and $R$ such that $\left\|S_{R}(\alpha) f\right\|_{p} \leq C\|f\|_{p}$ for all $f$ in $L^{p}$. As $R$ tends to infinity, $S_{R}(\alpha) f$ converges to $f$ in the norm.

Proof. The proof is very simple. Since $\alpha>1 / 6, E_{R}(x)$ is an $L^{1}$ function. Therefore, it follows immediately that $\left\|E_{R} f\right\|_{p} \leq C\|f\|_{p}$. This proves the 
uniform boundedness. For a $C^{\infty}$ function with compact support it is clear that $S_{R}(\alpha) f$ converges to $f$ in the norm. In view of the uniform boundedness of $S_{R}(\alpha)$, a density argument shows that $S_{R}(\alpha) f$ converges to $f$ in the norm. Hence the theorem.

Let us now recall a few facts about the Hardy-Littlewood maximal function defined as follows:

$$
\Lambda f(x)=\sup _{h>0}(2 h)^{-1} \int_{-h \leq y \leq h}|f(x-y)| d y .
$$

For $1<p<\infty, \Lambda$ is bounded on $L^{p}$. When $p=1$, we have the following weak type inequality:

$$
|\{x: \Lambda f(x)>\lambda\}| \leq C \lambda^{-1}\|f\|_{1} .
$$

Many maximal functions are dominated by the Hardy-Littlewood maximal function. One such maximal function is given by the following. Given $g$ in $L^{1}$ and $\varepsilon>0$, we can consider the family $\left\{g_{\varepsilon} * f\right\}$. Then we have $\sup _{\varepsilon>0}\left|g_{\varepsilon} * f(x)\right|$ $\leq C \Lambda f(x)$. In particular, this applied to $E_{R}(x)$ gives

$$
\sup _{R>0}\left|E_{R} f(x)\right| \leq C \Lambda f(x) .
$$

The following theorem will be used in the proof of the next theorem. See Stein [23] for a proof.

Theorem. Let $T_{\varepsilon}, \varepsilon>0$, be a family of linear operators mapping $L^{p}$ functions into measurable functions. For each $h$ in $L^{p}$ define $M h(x)=\sup _{\varepsilon>0}\left|T_{\varepsilon} f(x)\right|$. Suppose there is a constant $a>0$ and $a q \geq 1$ such that $|\{x: M h(x)>1\}| \leq$ $\left(a \lambda^{-1}\|f\|_{p}\right)^{q}$. If there is a dense subset $D$ of $L^{p}$ such that $\lim T_{\varepsilon} g(x)$ exists and is finite a.e. whenever $g$ is in $D$, then for each $f$ in $L^{p} \lim T_{\varepsilon} f(x)$ exists and is finite almost everywhere.

Consider the family of operators $\left\{S_{R}(\alpha)\right\}$. Let $M$ be the associated maximal function, i.e. $M f(x)=\sup _{R>0}\left|S_{R}(\alpha) f(x)\right|$. For this maximal function we have the following theorem.

Theorem 5.2. Assume that $\alpha>1 / 6$. There is a constant $C>0$ such that the following hold:

$$
\begin{aligned}
& \|M f\|_{p} \leq C\|f\|_{p}, \text { for all } f \text { in } L^{p}, 1<p<\infty, \\
& |\{x: M f(x)>\lambda\}| \leq C \lambda^{-1}\|f\|_{1}, \text { for all } f \text { in } L^{1} .
\end{aligned}
$$

We also have a.e. convergence: for $f$ in $L^{p}, 1 \leq p \leq \infty, S_{R}(\alpha) f$ converges to $f$ a.e. as $R \rightarrow \infty$.

Proof. In view of the foregoing observations it is clear that

$$
M f(x) \leq C\left\{\Lambda f(x)+\Lambda f^{*}(x)\right\} .
$$


(5.6) and (5.7) follow from the boundedness properties of the Hardy-Littlewood maximal function. The a.e. convergence follows from (5.6) and (5.7) by means of the above theorem.

Next we proceed to prove the analogues of the Fejer-Lebesgue theorem and Riemann's localisation principle. The following arguments are taken from Peetre [20]. See Hormander [11] also. We will get an estimate for $E_{R} f(x)$ when $f(y)$ vanishes for $|x-y|<r<1$. By Hölder's inequality we have $\left|E_{R} f(x)\right| \leq C R^{1 / 2}\left(1+R^{1 / 2} r\right)^{-\alpha-5 / 6}\|f\|_{1}$. We will first prove

Proposition 5.1. Assume that $\alpha>1 / 6$ and $f$ belongs to $L^{p}, 1 \leq p \leq \infty$. Then we have

$$
\left|E_{R} f(x)\right| \leq C\left\{\|f\|_{p}+\sup _{0<r<1}\left(r^{-1} \int_{|x-y|<r}|f(y)| d y\right)\right\} .
$$

Proof. Let $f_{0}(y)=f(y)$ for $|x-y|>1$ and 0 otherwise. For $k \geq 1$, we define $f_{k}(y)$ by setting

$$
f_{k}(y)=f(y) \text { for } 2^{-k} \leq y \leq 2^{-k+1} \text { and } 0 \text { otherwise. }
$$

Then in view of the above observations we have

$$
\begin{aligned}
\left|E_{R} f(x)\right| & \leq \sum_{k \geq 0}\left|E_{R} f_{k}(x)\right| \\
& \leq C\left\{\|f\|_{p}+R^{1 / 2} \sum_{k \geq 1}\left(1+2^{-k} R^{1 / 2}\right)^{-\alpha-5 / 6}\left\|f_{k}\right\|_{1}\right\} .
\end{aligned}
$$

If we set $F=\sup _{0<r<1}\left(r^{-1} \int_{|x-y|<r}|f(y)| d y\right)$ we obtain $\left\|f_{k}\right\|_{1} \leq C F 2^{-k}$ so the sum can be estimated by $C F \sum_{k \geq 1} 2^{-k} R^{1 / 2}\left(1+2^{-k} R^{1 / 2}\right)^{-\alpha-5 / 6}$. The sum converges even when it is extended from $-\infty$ to $\infty$, provided $\alpha>1 / 6$. It is then a bounded function of $R$ for it is clearly locally bounded and it remains unchanged if $R$ is replaced by $2^{2 k} R$. Hence we obtain the estimate $\left|E_{R} f(x)\right| \leq$ $C\left\{\|f\|_{p}+F\right\}$.

We recall one more definition. A point $x$ is said to be a Lebesgue point of an $L^{p}$ function $f$ if the following holds:

$$
\lim _{r \rightarrow 0} r^{-1} \int_{|x-y|<r}|f(x)-f(y)| d y=0 .
$$

We can now state and prove

Theorem 5.3 (Fejér-Lebesgue). Assume that $\alpha>1 / 6$ and $f$ belongs to $L^{p}$, $1 \leq p \leq \infty$. If $x$ and $-x$ are both Lebesgue points of $f$ then $S_{R}(\alpha) f(x)$ converges to $f(x)$ as $R$ tends to infinity.

Proof. The theorem is clearly true for $C^{\infty}$ functions with compact support. Replacing, if necessary, $f(y)$ by $f(y)-f(x) g(y)-f(-x) g(-y)$ where $g$ is a 
$C^{\infty}$ function such that $g(x)=1$ and $g(-x)=0$ we can assume that $f(x)=0$ and $f(-x)=0$. Thus, we are given that

$$
\begin{aligned}
& \lim _{r \rightarrow 0} r^{-1} \int_{|x-y|<r}|f(y)| d y=0, \\
& \lim _{r \rightarrow 0} r^{-1} \int_{|x+y|<r}|f(y)| d y=0 .
\end{aligned}
$$

Let $B$ be the Banach space of measurable functions with the norm

$$
\|f\|_{B}=\|f\|_{p}+\sup _{0<r<1}\left(r^{-1} \int_{|x-y|<r}|f(y)| d y\right)+\sup _{0<r<1}\left(r^{-1} \int_{|x+y|<r}|f(y)| d y\right) .
$$

Every $f$ in $L^{p}$ satisfying (i) and (ii) belongs to the closure of $B \cap C_{0}$ and $S_{R}(\alpha) f(x)$ converges to $f(x)$ for such functions. Therefore, we only have to show that $\sup _{R}\left\|S_{R}(x, y)\right\|_{B^{*}}<\infty$, where $\|g\|_{B^{*}}$ is the norm dual to $\|\cdot\|_{B}$ which is given by $\|g\|_{B^{*}}=\sup \left|\int g(y) f(y) d y\right|$ where the sup is taken over all $f$ with $\|f\|_{B} \leq 1$. But in view of Proposition 5.1 we immediately obtain $\left|\int S_{R}(x, y) f(y) d y\right| \leq C\|f\|_{B}$. Hence the theorem.

We remark that in the classical Fejer-Lebesgue theorem for the Fourier series it was only required that $x$ is a Lebesgue point of $f$. A similar remark applies to the following Riemann's localisation principle. As opposed to the case of the Fourier series where the vanishing of $f$ is assumed only near $x$, now we have to assume that the function vanishes near $-x$ as well.

Theorem 5.4 (Riemann's localisation principle). Assume that $\alpha>1 / 6$ and $f$ is in $L^{p}, 1 \leq p \leq \infty$. If $f$ vanishes near $x$ and $-x$, then $S_{R}(\alpha) f(x)$ converges to 0 as $R$ tends to infinity.

Proof. The proof is similar to that of Theorem 5.3. We consider the Banach space $E$ of measurable functions $f$ which vanish for $|x-y|<r$ and $|x+y|<$ $r, r$ is a fixed number, with the norm defined by

$$
\|f\|_{E}=\left(\int_{|x-y|>r}|f(y)| d y\right)+\left(\int_{|x+y|>r}|f(y)| d y\right)
$$

and proceed as in the previous theorem.

Finally we consider the summability below the critical index. The case $\alpha=0$ was settled by Askey and Wainger in [3]. They proved that the partial sum operators $S_{n}$ of the Hermite expansion are uniformly bounded on $L^{p}$ if and only if $4 / 3<p<4$. To settle the case $0<\alpha<1 / 6$ we use the complex interpolation methods of Stein.

Consider a family $T_{z}$ of linear operators depending on a complex parameter $z$. Assume that the following three conditions are satisfied: (1) for each $z$, $0<\operatorname{Re} z<1, T_{z}$ is a linear transformation of simple functions into measurable functions, (2) when $\varphi$ and $\psi$ are simple functions then the function $\Phi(z)=$ $\int T_{z} \psi(x) \varphi(x) d x$ is analytic in $0<\operatorname{Re} z<1$ and continuous in $0 \leq \operatorname{Re} z \leq 1$, 
and (3) $\sup _{|y| \leq r} \sup _{0 \leq x \leq 1} \log |\Phi(x+i y)| \leq A e^{a r}, a<\pi ; A$ and $a$ may depend on $\varphi$ and $\psi$. For such a family Stein [25] proved the following result.

Theorem (Stein). Suppose that $1 \leq p_{1}, p_{2}, q_{1}, q_{2} \leq \infty$ and that $1 / p=$ $(1-t) / p_{1}+t / p_{2}, 1 / q=(1-t) / q_{1}+t / q_{2}$ where $0 \leq t \leq 1$. Assume that whenever $f$ is simple the following inequalities hold:

$$
\begin{gathered}
\left\|T_{i y}(f)\right\|_{q_{1}} \leq A_{0}(y)\|f\|_{p_{1}}, \\
\left\|T_{1+i y}(f)\right\|_{q_{2}} \leq A_{1}(y)\|f\|_{p_{2}} .
\end{gathered}
$$

Suppose further that $\log A_{i}(y) \leq A e^{a|y|}, a<\pi$ for $i=1,2$. Then we have $\left\|T_{t}(f)\right\|_{q} \leq A_{t}\|f\|_{p}$.

We can now prove the following theorem regarding the uniform boundedness of $S_{R}(\alpha)$ when $\alpha$ is smaller than $1 / 6$.

Theorem 5.5. Assume that $0<\alpha<1 / 6$ and $f$ belongs to $L^{p}$. Then $S_{R}(\alpha)$ are uniformly bounded on $L^{p}$ if and only if $4 /(6 \alpha+3)<p<4 /(1-6 \alpha)$.

Proof. The only if part has been already proved in Theorem 2.1. To get the if part we interpolate between the results $\alpha=0$ and for $\alpha>1 / 6$. Consider the family of operators $T_{z} f$ defined by $T_{z} f=S_{R}(\alpha(z)) f$ where $\alpha(z)=(1 / 6+\varepsilon) z$, $\varepsilon>0$. That this family satisfies the conditions of Stein's theorem can be verified as in Hormander [11]. Applying Stein's theorem and then letting $\varepsilon$ tend to 0 we get the theorem.

\section{REFERENCES}

1. R. Askey, Norm inequalities for some orthogonal series, Bull. Amer. Math. Soc. 72 (1966), 803-823.

2. R. Askey and I. I. Hirschman, Jr., Mean summability for ultraspherical polynomials, Math. Scand. 12 (1963), 167-177.

3. R. Askey and S. Wainger, Mean convergence of expansions in Laguerre and Hermite series, Amer. J. Math. 87 (1965), 695-708.

4. R. Campbell, Determination effective de toutes les moyennes de Cesàro d'ordre entier pour les series de polynomes orthogonaux comprenant ceux de Laguerre et de Hermite, C.R. Acad. Sci. Paris 243 (1956), 882-885.

5. A. Erdelyi et al., Asymptotic forms for Laguerre polynomials, Golden Jubilee volume of the Indian Math. Soc.

6. D. Ernst, Über die konvergenz der $(C, 1)$-Mittel von Fourier-Laguerre-Riehen, Compositio Math. 21 (1969), 81-101.

7. G. Freud, On weighted simultaneous polynomial approximation, Studia Sci. Math. Hungar. 7 (1972), 337-342.

8. __ On polynomial approximation with respect to general weights, Functional Analysis and its Applications, Lecture Notes in Math., vol. 399, Springer-Verlag, Berlin and New York, 1974, pp. 149-179.

9. G. Freud and S. Knapowsky, On linear processes of approximation. III, Studia Math. 25 (1965), 373-383.

10. J. J. Gergen, Summability of double Fourier series, Duke Math. J. 3 (1937), 133-148. 
11. L. Hormander, On the Riesz means of spectral functions and eigenfunction expansions for elliptic differential operators, Some Recent Advances in the Basic Sciences, Yeshiva Univ., New York, 1966.

12. E. Kogbetliantz, Sur les moyennes arithmetiques des series-noyaux des developpement en series d'Hermite et de Laguerre et sur celles de les series-noyaux drivees term a terme, J. Math. Phys. 14 (1935), 37-99.

13. L. Lorch, The Lebesgue constants for Jacobi series. I, Proc. Amer. Math. Soc. 10 (1959), 756761.

14. C. Markett, Norm estimates for Cesàro means of Laguerre expansions, Proc. Conf. on Approximations and Function Spaces, Gdansk, 1979, North-Holland, Amsterdam, 1981, pp. 419-435.

15. __, Mean Cesaro summability of Laguerre expansions and norm estimates with shifted parameter, Anal. Math. 8 (1982), 19-37.

16. _ Norm estimates for $(C, \delta)$ means of Hermite expansions and bounds for $\delta_{\mathrm{eff}}$, Acta Math. Hungar. 43 (1984), 187-198.

17. B. Muckenhoupt, Mean convergence of Hermite and Laguerre series. I, Trans. Amer. Math. Soc. 147 (1970), 419-431.

18. _ Mean convergence of Hermite and Laguerre series. II, Trans. Amer. Math. Soc. 147 (1970), 433-460.

19. F. Olver, Asymptotics and special functions, Academic Press, New York, 1974.

20. J. Peetre, Remark on eigenfunction expansions for elliptic operators with constant coefficients, Math. Scand. 15 (1964), 83-92.

21. E. L. Poiani, Mean Cesàro summability of Laguerre and Hermite series, Trans. Amer. Math. Soc. 173 (1972), 1-31.

22. H. Pollard, The mean convergence of orthogonal series. I, Trans. Amer. Math. Soc. 62 (1947), 387-403.

23. E. M. Stein, Singular integrals and differentiability properties of functions, Princeton Univ. Press, Princeton, N.J., 1971.

24. __ Beijing lectures on Fourier analysis, Ann. of Math. Studies, no.112, Princeton Univ. Press, Princeton, N.J., 1986.

25. E. M. Stein and G. Weiss, Introduction to Fourier analysis on Euclidean spaces, Princeton Univ. Press, Princeton, N.J., 1971.

26. G. Szego, Orthogonal polynomials, Amer. Math. Soc. Colloq. Publ., Amer. Math. Soc., Providence, R.I., 1967.

Department of Mathematics, Princeton University, Princeton, New Jersey 08544

Taba Institute of Fundamental Research Centre, indian Institute of Science Campus, BANGALORE, INDIA (Current address) 